\title{
New Expression for the Functional Transformation of the Vector Cramér-Rao Lower Bound
}

\author{
Ali A. Nasir ${ }^{\dagger}$, Hani Mehrpouyan ${ }^{\ddagger}$, and Rodney A. Kennedy ${ }^{\dagger}$, \\ ${ }^{\dagger}$ Research School of Information Sciences and Engineering, the Australian National University, Australia. \\ $\ddagger$ Department of Electrical and Computer Engineering and Computer Science, California State University, Bakersfield, USA. \\ email: ali.nasir@anu.edu.au, hani.mehr@ieee.org, and rodney.kennedy@anu.edu.au
}

\begin{abstract}
Assume that it is desired to estimate $\alpha=\mathbf{f}(\theta)$, where $\mathbf{f}(\cdot)$ is an $r$-dimensional function. This paper derives the general expression for the functional transformation of the vector Cramér-Rao lower bound (CRLB). The derived bound is a tight lower bound on the estimation of uncoupled parameters, i.e., parameters that can be estimated separately. Unlike previous results in the literature, this new expression is not dependent on the inverse of the Fisher's information matrix (FIM) of the untransformed parameters, $\theta$. Thus, it can be applied to scenarios where the FIM for $\theta$ is ill-conditioned or singular. Finally, as an application, the derived transformation is applied to determine the exact CRLB for estimation of channel parameters in amplify-and-forward relaying networks.
\end{abstract}

Index Terms-Cramér-Rao lower bound (CRLB), Fisher information matrix (FIM), channel estimation.

\section{INTRODUCTION}

The Cramer-Rao lower bound (CRLB) provides a lower bound on the variance of unbiased estimators, and is widely used in the fields of communication and signal processing [1]. It frequently occurs in practice that the parameters that need to be estimated are a function of some more fundamental system parameters, $\boldsymbol{\theta}$. We refer to those required estimation parameters as transformed system parameters, $\boldsymbol{\alpha}=$ $\mathbf{f}(\boldsymbol{\theta})$. The final Cramér-Rao lower bound (CRLB) expression for the transformed system parameters has been well studied for real [1] and complex parameters [2]. Subsequently, the CRLB results in [2] were simplified in [3], and extended for the estimation of constrained parameters in [4] and for a combination of constrained and unconstrained parameters in [5]. Recently, the asymptotic behavior (high signal-to-noise ratio (SNR) and/or large number of samples) of the CRLB was investigated in [6]. Note that the final expression of the CRLB for transformed system parameters given in [1][6] depends on the inverse of the Fisher information matrix (FIM) of the fundamental system parameters, $\boldsymbol{\theta}$. However, it may happen that the FIM of these parameters is rank deficient or ill-conditioned implying that the CRLB expression is infinity or numerically unstable [7], even when an unbiased estimator and CRLB both exist. In such situations, it has been proposed to take the reciprocal of the corresponding

This research was supported under Australian Research Council's Discovery Projects funding scheme (project number DP110102548). diagonal elements of FIM [8]. However, the resulting bound is either loose for estimating untransformed system parameters [8] or incorrect for the estimation of transformed system parameters, as shown in this paper. Later, the use of pseudo inverse of the FIM has been proposed for such problems [7]. However, the calculation of pseudo inverse may be numerically unstable and computationally prohibitive as the dimension of an estimation problem increases.

In order to address this deficiency in the existing CRLB expressions, this letter derives a new CRLB expression for the estimation of transformed system parameters, which does not depend on any type of matrix inverse and is valid even when the FIM of the fundamental system parameters is rank deficient. The application and usefulness of the proposed result is demonstrated through its use in channel estimation for amplify-and-forward (AF) cooperative relaying networks. More specifically our problem concerns the estimation of an overall channel gain vector, $\boldsymbol{\alpha}$ from source-to-relaysto-destination (transformed system parameters), which is a function of individual channels from source-to-relays, $\mathbf{h}$, and from relays-to-destination, $\mathrm{g}$. These component channels are the fundamental system parameters or vector, i.e., $\boldsymbol{\theta} \triangleq$ $[\mathbf{h}, \mathbf{g}]^{T}$. In context of this channel estimation problem, the existing CRLB results have ambiguity about formulating the vector of parameters of interest, i.e., whether the derivative of $\mathbf{g}$ with respect to $\boldsymbol{\alpha}$ is zero or not (as can be observed in [9] or [10], respectively). Thus, the evaluation of direct CRLB is not obvious. Moreover, in the considered estimation problem, the FIM of fundamental system parameters, $\boldsymbol{\theta}$, is rank deficient. Hence in this situation no conclusions can be drawn regarding whether there exists an unbiased estimator and CRLB for estimation of the overall channel gains $\boldsymbol{\alpha}=\mathbf{f}(\boldsymbol{\theta})$. In contrast, as corroborated using a minimum variance unbiased (MVU) estimator, the CRLB expression in this letter, is numerically stable since it does not depend on any type of matrix inverse.

Notation: Superscripts $(\cdot)^{*},(\cdot)^{T},(\cdot)^{H}$, and $(\cdot)^{\dagger}$ denote the conjugate, the transpose, and the conjugate transpose, and Moore-Penrose pseudoinverse operators, respectively. $\odot$ and $\oslash$ are used for element-wise product and elementwise division of two vectors, respectively. $\mathbb{E}\{\cdot\}$ denotes the expectation operator. $\Re\{\cdot\}$ and $\Im\{\cdot\}$ denote the real and 
imaginary parts of a complex quantity. $\hat{x}$, represents the estimated value of $x \cdot \mathcal{C N}\left(\mu, \sigma^{2}\right)$ denotes a complex Gaussian distributions with mean $\mu$ and variance $\sigma^{2}$. Boldface small letters, $\mathbf{x}$, and boldface capital letters, $\mathbf{X}$, are used for vectors and matrices, respectively. $[\mathbf{x}]_{y}$ represents the $y$ th element of vector $\mathbf{x}$ and $[\mathbf{X}]_{x, y}$ represents the entry in row $x$ and column $y$ of $\mathbf{X} . \mathbf{I}_{X}$ and $\mathbf{0}_{X \times X}$ denote the $X \times X$ identity and all zero matrices. $\operatorname{Tr}\{\mathbf{X}\}$ denotes the trace of $\mathbf{X}$. $\operatorname{diag}(\mathbf{x})$ is used to denote a diagonal matrix, where its diagonal elements are given by the vector $\mathbf{x}$. Finally, a set of complex $x$ dimensional vectors is denoted by $\mathbb{C}^{x}$ and $\mathbf{f}: \mathbb{C}^{x} \rightarrow \mathbb{C}^{y}$ is used to represent that the function $\mathbf{f}$ takes as its argument a complex $x$-dimensional vector and returns a complex $y$ dimensional vector.

\section{Functional Transformation of Vector CRLB}

Assume that it is desired to estimate $\boldsymbol{\alpha}=\mathbf{f}(\boldsymbol{\theta})$, where $\boldsymbol{\theta} \in \mathbb{C}^{p}, \boldsymbol{\alpha} \in \mathbb{C}^{r}$, and $\mathbf{f}: \mathbb{C}^{p} \rightarrow \mathbb{C}^{r}$. Using functional transformation of the vector CRLB in [2], [7], the CRLB for the estimation of $\alpha_{k}=[\mathbf{f}(\boldsymbol{\theta})]_{k}$, for $k=1, \ldots, r$, is given by

$$
\begin{aligned}
\operatorname{CRLB}_{\alpha_{k}} & =\frac{\partial[\mathbf{f}(\boldsymbol{\theta})]_{k}}{\partial \boldsymbol{\theta}^{T}} \mathbf{F}_{\boldsymbol{\theta}}^{-1}\left(\frac{\partial[\mathbf{f}(\boldsymbol{\theta})]_{k}}{\partial \boldsymbol{\theta}^{T}}\right)^{H}, \\
\mathrm{CRLB}_{\alpha_{k}} & =\frac{\partial[\mathbf{f}(\boldsymbol{\theta})]_{k}}{\partial \boldsymbol{\theta}^{T}} \mathbf{F}_{\boldsymbol{\theta}}^{\dagger}\left(\frac{\partial[\mathbf{f}(\boldsymbol{\theta})]_{k}}{\partial \boldsymbol{\theta}^{T}}\right)^{H},
\end{aligned}
$$

where $\mathbf{F}_{\boldsymbol{\theta}}$ is the FIM of the untransformed parameter, $\boldsymbol{\theta}$. If $\mathbf{F}_{\boldsymbol{\theta}}$ is singular or ill-conditioned, the bound in (1a) results in an invalid CRLB for estimation of $\alpha_{k}$. Moreover, the bound in (1b) can also result in an invalid CRLB due to numerical instability, e.g., due to quantization error or can be computationally prohibitive to evaluate, e.g., if the dimension of $\boldsymbol{\theta}$ is significantly larger than $\boldsymbol{\alpha}$. To address these shortcomings, here, a new expression for calculating the CRLB of transformed parameter, $\boldsymbol{\alpha}$, that does not require the evaluation of any form of matrix inverse is derived.

Let us assume that the likelihood function, $p(\mathbf{y} ; \boldsymbol{\theta})$, satisfies the regularity condition [1], i.e.,

$$
\mathbb{E}\left\{\frac{\partial \ln p(\mathbf{y} ; \boldsymbol{\theta})}{\partial \boldsymbol{\theta}}\right\}=\mathbf{0}_{p \times 1}, \quad \forall \boldsymbol{\theta},
$$

where $p(\mathbf{y} ; \boldsymbol{\theta})$ is the likelihood function of the complex observation vector, $\mathbf{y}$, parameterized by $\boldsymbol{\theta}$ and expectation is taken with respect to $p(\mathbf{y} ; \boldsymbol{\theta})$. For an unbiased estimator,

$$
\mathbb{E}\{\hat{\boldsymbol{\alpha}}\}=\boldsymbol{\alpha}=\mathbf{f}(\boldsymbol{\theta}) .
$$

Using (2) and (3), we have

$$
\int(\hat{\boldsymbol{\alpha}}-\boldsymbol{\alpha}) \frac{\partial \ln p(\mathbf{y} ; \boldsymbol{\theta})}{\partial \boldsymbol{\theta}^{T}} p(\mathbf{y} ; \boldsymbol{\theta}) d \mathbf{y}=\frac{\partial \mathbf{f}(\boldsymbol{\theta})}{\partial \boldsymbol{\theta}^{T}} .
$$

Pre- and post-multiplying (4) by $\mathbf{a}^{T}$ and $\mathbf{b}$, respectively, where $\mathbf{a}$ and $\mathbf{b}$ are arbitrary $r \times 1$ and $p \times 1$ vectors, we have

$$
\int \mathbf{a}^{T}(\hat{\boldsymbol{\alpha}}-\boldsymbol{\alpha}) \frac{\partial \ln p(\mathbf{y} ; \boldsymbol{\theta})}{\partial \boldsymbol{\theta}^{T}} p(\mathbf{y} ; \boldsymbol{\theta}) \mathbf{b} d \mathbf{y}=\mathbf{a}^{T} \frac{\partial \mathbf{f}(\boldsymbol{\theta})}{\partial \boldsymbol{\theta}^{T}} \mathbf{b} .
$$

Applying Cauchy-Schwarz inequality [1, p. 71]

$$
\begin{aligned}
& \mid \int m(\mathbf{y})\left.n(\mathbf{y}) o(\mathbf{y}) d \mathbf{y}\right|^{2} \\
& \leq \int m(\mathbf{y})|n(\mathbf{y})|^{2} d \mathbf{y} \int m(\mathbf{y})|o(\mathbf{y})|^{2} d \mathbf{y} .
\end{aligned}
$$

where $m(\mathbf{y}) \triangleq p(\mathbf{y} ; \boldsymbol{\theta}), n(\mathbf{y}) \triangleq \mathbf{a}^{T}(\hat{\boldsymbol{\alpha}}-\boldsymbol{\alpha})$, and $o(\mathbf{y}) \triangleq$ $\frac{\partial \ln p(\mathbf{y} ; \boldsymbol{\theta})}{\partial \boldsymbol{\theta}^{T}} \mathbf{b}$. Thus, (5) can be written as (7) given at the bottom of this page, where $\boldsymbol{\Sigma}_{\hat{\alpha}}$ is the covariance of $\hat{\boldsymbol{\alpha}}$. Let $\mathbf{b}=\left(\frac{\partial \mathbf{f}(\boldsymbol{\theta})}{\partial \boldsymbol{\theta}^{T}}\right)^{H} \mathbf{a}$. Then, (7) can be written as

$$
\begin{aligned}
& \left|\mathbf{a}^{T} \frac{\partial \mathbf{f}(\boldsymbol{\theta})}{\partial \boldsymbol{\theta}^{T}}\left(\frac{\partial \mathbf{f}(\boldsymbol{\theta})}{\partial \boldsymbol{\theta}^{T}}\right)^{H} \mathbf{a}\right|^{2} \\
& \quad \leq \mathbf{a}^{T} \boldsymbol{\Sigma}_{\hat{\boldsymbol{\alpha}}} \mathbf{a}^{*} \mathbf{a}^{H} \frac{\partial \mathbf{f}(\boldsymbol{\theta})}{\partial \boldsymbol{\theta}^{T}} \mathbf{F}_{\boldsymbol{\theta}}\left(\frac{\partial \mathbf{f}(\boldsymbol{\theta})}{\partial \boldsymbol{\theta}^{T}}\right)^{H} \mathbf{a} .
\end{aligned}
$$

In order to find the lower bound on the estimation $\alpha_{k}=$ $[\mathbf{f}(\boldsymbol{\theta})]_{k}$ for $k=1, \ldots, r$, let $\mathbf{a}=\left[\mathbf{0}_{1 \times k-1}, 1, \mathbf{0}_{1 \times r-k}\right]^{T}$. Thus, (8) can be written as

$$
\left|\frac{\partial[\mathbf{f}(\boldsymbol{\theta})]_{k}}{\partial \boldsymbol{\theta}^{T}}\left(\frac{\partial[\mathbf{f}(\boldsymbol{\theta})]_{k}}{\partial \boldsymbol{\theta}^{T}}\right)^{H}\right|^{2} \leq \sigma_{\hat{\alpha}_{k}}^{2} \frac{\partial[\mathbf{f}(\boldsymbol{\theta})]_{k}}{\partial \boldsymbol{\theta}^{T}} \mathbf{F}_{\boldsymbol{\theta}}\left(\frac{\partial[\mathbf{f}(\boldsymbol{\theta})]_{k}}{\partial \boldsymbol{\theta}^{T}}\right)^{H},
$$

where $\sigma_{\hat{\alpha}_{k}}^{2}=\left[\boldsymbol{\Sigma}_{\hat{\alpha}}\right]_{k, k}$ is the variance of $\hat{\alpha}_{k}$. Subsequently, $\sigma_{\hat{\alpha}_{k}}^{2}$ is given by

$$
\sigma_{\hat{\alpha}_{k}}^{2} \geq \frac{\left|\frac{\partial[\mathbf{f}(\boldsymbol{\theta})]_{k}}{\partial \boldsymbol{\theta}^{T}}\left(\frac{\partial[\mathbf{f}(\boldsymbol{\theta})]_{k}}{\partial \boldsymbol{\theta}^{T}}\right)^{H}\right|^{2}}{\frac{\partial[\mathbf{f}(\boldsymbol{\theta})]_{k}}{\partial \boldsymbol{\theta}^{T}} \mathbf{F}_{\boldsymbol{\theta}}\left(\frac{\partial[\mathbf{f}(\boldsymbol{\theta})]_{k}}{\partial \boldsymbol{\theta}^{T}}\right)^{H}}, \quad k=1, \ldots, r .
$$

Using (9), the CRLB for the estimation of all the elements of the transformed parameter vector $\boldsymbol{\alpha}$ can be obtained.

Remark 1: Unlike the CRLB expressions for the transformed parameters in (1) that rely on the inverse or pseudo inverse of FIM of the untransformed parameters, the transformed CRLB in (10) does not depend on any type of inverse of the FIM of untransformed parameters, $\boldsymbol{\theta}$. Thus, the proposed functional transformation of vector CRLB in (10) results in a valid lower bound for $\boldsymbol{\alpha}$ regardless of whether the FIM of the untransformed parameter is singular or not (see Section III-C for more details). It is also important to note that following (10), we are able to find the diagonal

$$
\begin{aligned}
\left|\mathbf{a}^{T} \frac{\partial \mathbf{f}(\boldsymbol{\theta})}{\partial \boldsymbol{\theta}^{T}} \mathbf{b}\right|^{2} & \leq \int p(\mathbf{y} ; \boldsymbol{\theta}) \mathbf{a}^{T}(\hat{\boldsymbol{\alpha}}-\boldsymbol{\alpha})(\hat{\boldsymbol{\alpha}}-\boldsymbol{\alpha})^{H} \mathbf{a}^{*} d \mathbf{y} \int p(\mathbf{y} ; \boldsymbol{\theta}) \mathbf{b}^{H}\left(\frac{\partial \ln p(\mathbf{y} ; \boldsymbol{\theta})}{\partial \boldsymbol{\theta}^{T}}\right)^{H} \frac{\partial \ln p(\mathbf{y} ; \boldsymbol{\theta})}{\partial \boldsymbol{\theta}^{T}} \mathbf{b} d \mathbf{y} \\
& =\mathbf{a}^{T} \boldsymbol{\Sigma}_{\hat{\boldsymbol{\alpha}}} \mathbf{a}^{*} \mathbf{b}^{H} \mathbf{F}_{\boldsymbol{\theta}} \mathbf{b} .
\end{aligned}
$$


elements of the CRLB. Thus, the derived bound is a tight lower bound for the estimation of uncoupled parameters, i.e., parameters that do not need to be jointly estimated. A practical application of the derived CRLB for lower bounding channel estimation accuracy in AF cooperative relaying networks is presented in the following section.

\section{ApPlication of The PROPOSED CRLB}

In this section, as an application, the derived CRLB expression in (10) is used to obtain the lower bond on the channel estimation variance in a single-input-single-output (SISO) multi-relay cooperative network. The network consists of one source node, $K$ relays, and a single destination node. Quasi-static and frequency flat-fading channel parameters from source to the $k$ th relay and $k$ th relay to destination are denoted by $h_{k}$ and $g_{k}$, respectively, for $k=\{1, \ldots, K\}$. It is assumed that unit-amplitude phase shift keying (PSK) training signals (TSs) are transmitted from source to the $k$ th relay and from the $k$ th relay to the destination, $\forall k$.

The received complex baseband signal at the $k$ th relay, $\mathbf{r}_{k} \triangleq\left[r_{k}(0), \ldots, r_{k}(L-1)\right]^{T}$, is given by [11]

$$
\mathbf{r}_{k}=h_{k} \mathbf{t}^{[s]}+\mathbf{u}_{k},
$$

where $L$ is the length of observation vector, $\mathbf{t}^{[s]} \triangleq$ $\left[t^{[s]}(0), \ldots, t^{[s]}(L-1)\right]^{T}$ is the training signal from source to relays, $h_{k}$ is the unknown flat fading channel parameter, $\mathbf{u}_{k} \triangleq\left[u_{k}(0), \ldots, u_{k}(L-1)\right]^{T}$, and $u_{k}(i)$ for $i=0, \ldots, L-$ 1 , denotes the zero-mean complex additive white Gaussian noise (AWGN) at the $i$ th sample of the received signal at the $k$ th relay, i.e., $u_{k}(i) \sim \mathcal{C N}\left(0, \sigma_{u_{k}}^{2}\right)$. Each $k$ th relay applies distinct training signal $\mathbf{t}_{k}^{[r]} \triangleq\left[t_{k}^{[r]}(0), \ldots, t_{k}^{[r]}(L-1)\right]^{T}$ to the received signal [10]. The received signal at the destination, $\mathbf{y} \triangleq[y(0), \ldots, y(L-1)]^{T}$, is given by [11]

$$
\mathbf{y}=\mathbf{T} \boldsymbol{\alpha}+\mathbf{N} \boldsymbol{\beta}+\mathbf{w}
$$

where

- $\mathbf{T} \triangleq\left[\mathbf{t}_{1}, \ldots, \mathbf{t}_{K}\right]$ is an $L \times K$ matrix, $\mathbf{t}_{k}=\mathbf{t}^{[s]} \odot \mathbf{t}_{k}^{[r]}$, $\forall k$, is combined training from source and $k$ th relay,

- $\mathbf{N} \triangleq\left[\mathbf{n}_{1}, \ldots, \mathbf{n}_{K}\right]$ is an $L \times K$ matrix, $\mathbf{n}_{k}=\mathbf{u}_{k} \odot \mathbf{t}_{k}^{[r]}, \forall$ $k$, is the noise signal affected by the training signal from the $k$ th relay, such that the noise statistics of $\mathbf{n}_{k}$ and $\mathbf{u}_{k}$ are same, i.e., $n_{k}(i) \sim \mathcal{C N}\left(0, \sigma_{u_{k}}^{2}\right)$, for $i=0, \ldots, L-1$, due to unit amplitude PSK training signals,

- $\boldsymbol{\alpha} \triangleq\left[\alpha_{1}, \ldots, \alpha_{K}\right]^{T}$ is the overall channel gain from source to relay to destination such that $\alpha_{k}=\zeta_{k} h_{k} g_{k}$, $\zeta_{k}=1 / \sqrt{\sigma_{u_{k}}^{2}+\sigma_{h}^{2}}$ is the $k$ th relay's power constraint,

- $\boldsymbol{\beta} \triangleq\left[\beta_{1}, \ldots, \beta_{K}\right]^{T}$ such that $\beta_{k}=\zeta_{k} g_{k}$,

- $\mathbf{w} \triangleq[w(0), \ldots, w(L-1)]^{T}$, and $w(i)$ for $i=$ $0, \ldots, L-1$, denotes the zero-mean complex AWGN at the destination, i.e., $w(i) \sim \mathcal{C N}\left(0, \sigma_{w}^{2}\right)$.

The following subsection evaluates the CRLB expression for estimating transformed parameter $\boldsymbol{\alpha}$, which depends on the fundamental system parameters $\boldsymbol{\theta} \triangleq\left[\mathbf{h}^{T}, \mathbf{g}^{T}\right]^{T}$

\section{A. CRLB Evaluation}

Based on the assumptions in Section III, the AWGN at the relays, $\mathbf{u}_{k}, \forall k$, and destination, $\mathbf{w}$ in (12), are mutually independent. Accordingly, the received training signal at the destination, $\mathbf{y}$ in (12), is a circularly symmetric complex Gaussian random variable, $\mathbf{y} \sim \mathcal{C N}\left(\boldsymbol{\mu}_{\mathbf{y}}, \boldsymbol{\Sigma}_{\mathbf{y}}\right)$, with mean $\boldsymbol{\mu}_{\mathbf{y}}=\mathbf{T} \boldsymbol{\alpha}$ and covariance matrix $\boldsymbol{\Sigma}_{\mathbf{y}}=\left(\sum_{k=1}^{K} \sigma_{u_{k}}^{2}\left|\beta_{k}\right|^{2}+\right.$ $\left.\sigma_{w}^{2}\right) \mathbf{I}_{L}$. In order to find CRLB for the estimation of overall channel gain $\boldsymbol{\alpha}=\mathbf{f}(\boldsymbol{\theta})$, we can use the proposed CRLB expression in (10), where

$$
\frac{\partial \boldsymbol{\alpha}}{\partial \boldsymbol{\theta}^{T}}=[\operatorname{diag}(\boldsymbol{\zeta} \odot \mathbf{g}) \operatorname{diag}(\boldsymbol{\zeta} \odot \mathbf{h})],
$$

where $\zeta \triangleq\left[\zeta_{1}, \ldots, \zeta_{K}\right]^{T}$, and FIM for the untransformed parameter, $\boldsymbol{\theta} \triangleq\left[\mathbf{h}^{T}, \mathbf{g}^{T}\right]^{T}$, can be calculated using following expression [1], [12]

$$
\mathbf{F}_{\boldsymbol{\theta}}=\left(\frac{\partial \boldsymbol{\mu}_{\mathbf{y}}^{H}}{\partial \boldsymbol{\theta}^{*}} \boldsymbol{\Sigma}_{\mathbf{y}}^{-1} \frac{\partial \boldsymbol{\mu}_{\mathbf{y}}}{\partial \boldsymbol{\theta}^{T}}\right)+\operatorname{Tr}\left\{\boldsymbol{\Sigma}_{\mathbf{y}}^{-1} \frac{\partial \boldsymbol{\Sigma}_{\mathbf{y}}}{\partial \boldsymbol{\theta}^{*}} \boldsymbol{\Sigma}_{\mathbf{y}}^{-1} \frac{\partial \boldsymbol{\Sigma}_{\mathbf{y}}}{\partial \boldsymbol{\theta}^{T}}\right\} .
$$

Using the definition of $\boldsymbol{\mu}_{\mathbf{y}}$ and $\boldsymbol{\Sigma}_{\mathbf{y}}$, defined above (13), $\mathbf{F}_{\boldsymbol{\theta}}$ is given by

$$
\mathbf{F}_{\boldsymbol{\theta}}=\frac{1}{\rho}\left[\begin{array}{cc}
\mathbf{G}^{H} \mathbf{T}^{H} \mathbf{T G} & \mathbf{G}^{H} \mathbf{T}^{H} \mathbf{T H} \\
\mathbf{H}^{H} \mathbf{T}^{H} \mathbf{T G} & \mathbf{H}^{H} \mathbf{T}^{H} \mathbf{T H}+\operatorname{Tr}\left\{\boldsymbol{\Sigma}_{\mathbf{y}}^{-1} \boldsymbol{\Sigma}_{\mathbf{y}}^{-1}\right\} \boldsymbol{\epsilon} \epsilon^{H}
\end{array}\right],
$$

where $\mathbf{G} \triangleq \operatorname{diag}(\boldsymbol{\beta}), \mathbf{H} \triangleq \operatorname{diag}(\boldsymbol{\zeta} \odot \mathbf{h}), \rho \triangleq$ $\sum_{k=1}^{K} \sigma_{u_{k}}^{2}\left|\beta_{k}\right|^{2}+\sigma_{w}^{2}, \boldsymbol{\epsilon} \triangleq\left[\epsilon_{1}, \ldots, \epsilon_{K}\right]^{T}$, and $\epsilon_{k} \triangleq \sigma_{u_{k}}^{2} \zeta_{k}^{2} g_{k}$.

Remark 2: The FIM, $\mathbf{F}_{\boldsymbol{\theta}}$ in (15) is rank-deficient. Particularly, the rank of $2 K \times 2 K$ matrix $\mathbf{F}_{\boldsymbol{\theta}}$ is $K+1$. Thus, there is a rank deficiency of $K-1$ degrees. The rank of the matrix $\mathbf{F}_{\boldsymbol{\theta}}$ can be found by evaluating reduced row echelon form $\mathbf{U}$ of $\mathbf{F}_{\boldsymbol{\theta}}$, which is determined as

$$
\mathbf{U}=\left[\begin{array}{cc}
\mathbf{I}_{K} & \operatorname{diag}(\mathbf{h} \oslash \mathbf{g}) \\
\mathbf{0}_{1 \times K} & \mathbf{x}^{T} \\
\mathbf{0}_{K-1 \times K} & \mathbf{0}_{K-1 \times K}
\end{array}\right],
$$

where $\mathbf{x} \triangleq\left[x_{1}, \ldots, x_{K}\right]^{T}$ and $x_{k}=\frac{\sigma_{u_{k}}^{2} \zeta_{k}^{2} g_{k}^{*}}{\sigma_{u_{1}}^{2} \zeta_{1}^{2} g_{1}^{*}}$. The $K-1$ rows of zeros in $\mathbf{U}$ indicates the rank deficiency of $\mathbf{F}_{\boldsymbol{\theta}}$ by $K-1$ degrees. Thus, the existing CRLB expression for the evaluation of transformed system parameter in (1) results in an invalid bound. However, using (13) and (15), our proposed expression in (10) is able to calculate the CRLB of transformed parameter, $\alpha$, accurately (see Section III-C).

\section{B. MVU Estimation}

In this subsection, the best linear unbiased estimator (BLUE) for the estimation of $\boldsymbol{\alpha}$ is presented. Given that the received signal in (12) is Gaussian distributed, the BLUE can also be shown to be the MVU estimator [1, p. 133]. Following the approach given in $[1$, ch. 6], the BLUE or MVU estimate of $\boldsymbol{\alpha}$ is given by

$$
\hat{\boldsymbol{\alpha}}=\left(\mathbf{T}^{H} \mathbf{T}\right)^{-1} \mathbf{T}^{H} \mathbf{y},
$$

where the estimation covariance matrix of the linear unbiased estimate $\hat{\boldsymbol{\alpha}}$ is $\left(\mathbf{T}^{H} \boldsymbol{\Sigma}_{\mathbf{y}}^{-1} \mathbf{T}\right)^{-1}$. 


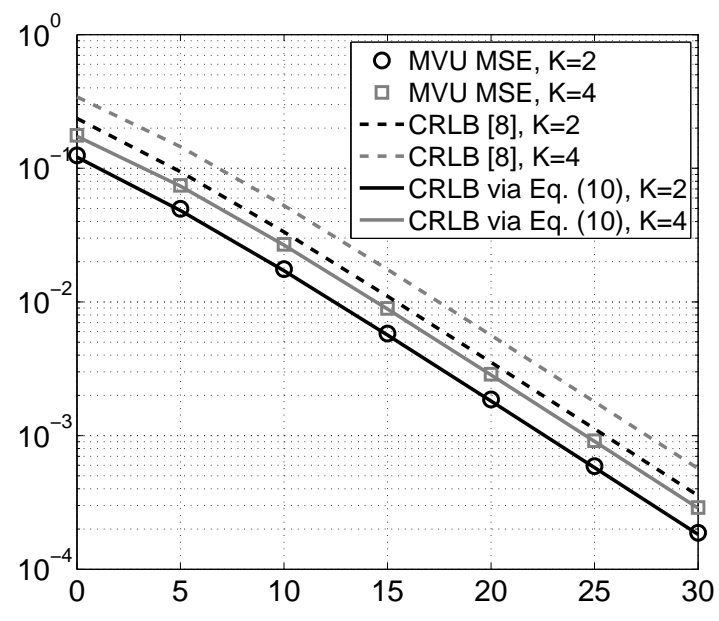

Fig. 1: MSE, proposed CRLB in (10), and the CRLB in [8], for the estimation of transformed parameter, $\alpha=$ $\mathbf{f}(\boldsymbol{\theta})$ for training length $L=16$ and different number of relays, $K=2$ and $K=4$.

\section{Numerical Simulation}

In this subsection, we numerically simulate the proposed CRLB in (10) and mean-square error (MSE) for the estimation of overall channel gain $\alpha$. Specific channels are used to evaluate the CRLB and MSE of MVU estimator, i.e., $\mathbf{h}=[.279-.9603 j, .8837+.4681 j,-.343+.732 i,-.734-$ $.451 i]^{T}$ and $\mathbf{g}=[.7820+.6233 j, .9474-.3203 j,-.2413+$ $.724 i, .5141-.893 i]^{T}$, similar to [13] and [10]. Note that the first two and all four entries of $\mathbf{h}$ and $\mathbf{g}$ are used for $K=2$ and $K=4$ relays, respectively. Distinct unit-amplitude PSK training signals are transmitted from source and different relays with training length $L=16$. It is assumed that the noise at all relays have the same variance, i.e., $\sigma_{u}^{2}=$ $\sigma_{u_{1}}^{2}=\cdots=\sigma_{u_{K}}^{2}$. Moreover, without loss of generality, it is assumed that $\sigma_{w}^{2}=\sigma_{u}^{2}=1 / \mathrm{SNR}$. The estimation MSE is numerically calculated as $\frac{1}{R} \sum_{r=1}^{R}\left(\alpha_{1}-\hat{\alpha}_{1}(r)\right)^{2}$, where $R=10000$ is the total number of simulations, $\hat{\alpha}_{1}(r)$ is the estimate of $\alpha_{1}$ during $r$ th simulation for $r=1, \ldots, R$. Without loss of generality, CRLB and estimation MSE for the first relay are plotted however similar results are obtained for all the relays.

Fig. 1 plots the CRLB and MSE for the estimation of $\boldsymbol{\alpha}$ versus SNR for $K=2$ and 4 relays. It can be observed from Fig. 1 that estimation MSE of MVU estimator is close to the proposed CRLB for the whole range of considered SNR values. The results in Fig. 1 also verifies the proposed CRLB expression in (10). On the other hand, the existing CRLB expressions in (1a) depends on the inverse of $\mathbf{F}_{\boldsymbol{\theta}}$, which is undefined for singular FIM. Fig. 1 also plots the CRLB in [8], where it has been proposed to replace $\mathbf{F}_{\boldsymbol{\theta}}^{-1}$ in (1a) with a diagonal matrix, whose diagonal elements are the reciprocal of the corresponding diagonal elements of $\mathbf{F}_{\boldsymbol{\theta}}$. It

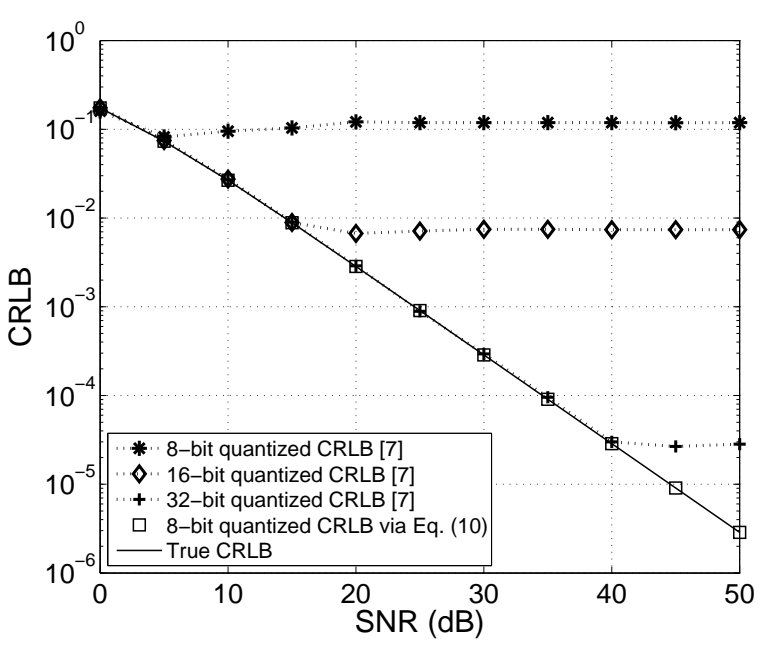

Fig. 2: Comparison of the proposed CRLB in (10) with [7] (see (1b)) for different levels of quantization. Other parameters are set to $K=4$ and $L=16$

can be observed from Fig. 1 that such bound is above the MSE of the proposed MVU estimator and is not a valid lower bound. ${ }^{1}$

Fig. 2 compares the proposed CRLB in (10) with [7] (see (1b)) for different levels of quantization. In order to investigate the numerical stability of the proposed expression in (10) over the existing result in (1b), 8, 16, and 32 bit quantization is applied. It can be observed from Fig. 2 that our proposed expression is not sensitive to quantization error and even produce the true CRLB with 8-bit quantization. ${ }^{2}$ However, the CRLB expression in (1b) is very sensitive to quantization error. This is because the CRLB expression in (1b) depends on the pseudo inverse of $\mathbf{F}_{\boldsymbol{\theta}}$, which is numerically unstable.

\section{CONCLUSION}

This letter derives the general CRLB expression for the estimation of transformed system parameters, $\boldsymbol{\alpha}=\mathbf{f}(\boldsymbol{\theta})$ which are a function of some fundamental system parameters, $\theta$. Unlike the previous results in the literature, the final CRLB expression, derived in this letter, does not depend on the inverse or pseudo inverse of the FIM of the untransformed parameters, $\boldsymbol{\theta}$. Thus, it can be applied to scenarios where the FIM of $\boldsymbol{\theta}$ is ill-conditioned/singular or when the pseudo inverse of the FIM of $\boldsymbol{\theta}$ is numerically unstable or computationally prohibitive to evaluate. Finally, as an example the proposed bound is applied in the context of channel estimation for in amplify-and-forward cooperative relaying

\footnotetext{
${ }^{1}$ According to [8], their CRLB should be loose bound for untransformed parameter estimation. However, for the estimation of transformed parameters, the CRLB in [8] is incorrect as demonstrated in Fig. 1.

${ }^{2}$ Similar results as true CRLB are obtained for 16 and 32 bit quantization with the proposed expression.
} 
networks, where shown that the MSE of an MVU estimator is close to the derived CRLB for wide range of SNR values.

\section{REFERENCES}

[1] S. M. Kay, Fundamentals of Statistical Signal Processing: Estimation Theory. NJ: Prentice Hall, 1993.

[2] A. van den Bos, "A Cramér-Rao lower bound for complex parameters," IEEE Trans. Signal Process., vol. 42, no. 10, p. 2859, Oct. 1994.

[3] S. T. Smith, "Statistical resolution limits and the complexified CramérRao bound," IEEE Trans. Signal Process., vol. 53, no. 5, pp. 15971609 , May 2005.

[4] A. K. Jagannatham and B. D. Rao, "Cramer-Rao lower bound for constrained complex parameters," IEEE Signal Process. Lett., vol. 11, no. 11, pp. 875-878, Nov. 2004.

[5] E. Ollila, V. Koivunen, and J. Eriksson, "On the Cramér-Rao bound for the constrained and unconstrained complex parameters," in Proc. IEEE Sensor Array and Multichannel Signal Processing Workshop. 2008, pp. 414-418

[6] T. Menni, E. Chaumette, P. Larzabal, and J. P. Barbot, "New results on deterministic Cramér-Rao bounds for real and complex parameters," IEEE Trans. Signal Process., vol. 60, no. 3, pp. 1032-1049, Mar. 2012
[7] P. Stoica and T. L. Marzetta, "Parameter estimation problems with singular information matrices," IEEE Trans. Signal Process., vol. 49 no. 1, pp. 87-0, Jan. 2001.

[8] B. Bobrovsky, E. M.-Wolf, and M. Zakai, "Some classes of global Cramer-Rao bounds," The Annals of Statistics, vol. 15, pp. 1421-1438, 1987.

[9] X. Li, C. Xing, Y.-C. Wu, and S. C. Chan, "Timing estimation and resynchronization for amplify-and-forward communication systems,' IEEE Trans. Signal Process., vol. 58, no. 4, pp. 2218-2229, Apr. 2010.

[10] H. Mehrpouyan and S. D. Blostein, "Bounds and algorithms for multiple frequency offset estimation in cooperative networks," IEEE Trans. Wireless Commun., vol. 10, no. 4, pp. 1300-1311, Apr. 2011.

[11] A. A. Nasir, H. Mehrpouyan, S. D. Blostein, S. Durani, and R. A. Kenedy, "Timing and carrier synchronization with channel estimation in multi-relay cooperative networks," IEEE Trans. Signal Process., vol. 60, no. 3, pp. 793-811, Mar. 2012.

[12] S. Zhang, F. Gao, and C.-X. Pei, "Optimal training design for individual channel estimation in two-way relay networks," IEEE Trans. Signal Process., vol. 60, no. 9, pp. 4987-4991, 20122012.

[13] T.-H. Pham, A. Nallanathan, and Y.-C. Liang, "Joint channel and frequency offset estimation in distributed MIMO flat-fading channels," IEEE Trans. Wireless Commun., vol. 7, no. 2, pp. 648-656, Feb. 2008. 